\title{
ON REGULAR SEMI-OPEN SETS AND S*-CLOSED SPACES
}

\author{
S. F. TADROS AND A. B. KHALAF
}

\begin{abstract}
In this note, the "egular semi-open sets, introduced in [2], are further investigated. Using covers consisting of such sets, a new class

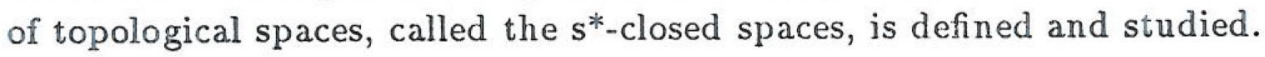

\section{Preliminaries}

By a space $(X, \tau)$ we mean a topological space on which no separation axiom is assumed. We recall the following definitions, notational conventions and characterizations. The closure (interior) of a subset $A$ of $X$ is denoted by $\mathrm{Cl} A$ (resp. Int $A$ ). $A$ is called regular open (regular closed) iff $A=\operatorname{IntCl} A$ (resp. $A=\mathrm{C} l \operatorname{Int} A)$. The family of all regular open (regular closed) subsets of $(X, \tau)$ is denoted by $R O(X, \tau)$ (resp. $R C(X, \tau)$ ). A set $A$ is said to be semi- $(\alpha-$, pre-, $\beta-$ ) open subset of $(X, \tau)$ iff $A \subset C$ ClInt $A$ [6] (resp. $A \subset \operatorname{IntClInt} A$ [9], $A \subset \operatorname{IntCl} A$ [8], $A \subset C / \operatorname{IntCl} A$ [1] ). The complement of each semi(resp. $\alpha-$, pre-, $\beta-$ ) open set is called a semi- (resp. $\alpha-$, pre-, $\beta-$ ) closed set. The family of all semi-open ( $\alpha$-open, pre-open, $\beta$-open, semi-closed, $\alpha$-closed, pre-closed, $\beta$-closed) subsets of $(X, \tau)$ is denoted by $S O(X, \tau)$ (resp. $\alpha O(X, \tau), P O(X, \tau), \beta O(X, \tau), S C(X, \tau), \alpha C(X, \tau), P C(X, \tau), \beta C(X, \tau))$. The semi-closure (semi-interior) of a set $A$, denoted by $s \mathrm{Cl} A$ (resp. $\operatorname{sint} A$ ), is defined in a natural way [3], $A \in S C(X, \tau)$ iff $s C l A=A$ and $A \in S O(X, \tau)$ iff

Received September 30, 1991.

AMS (MOS) subject classifications (1970). Primary 54D20, 54D30, 54D99; secondary $54 \mathrm{G} 05$. 
$s \operatorname{Irt} A=A$. It is known that $s \mathrm{C} l A=X \backslash s \operatorname{Int}(X \backslash A)[3], s \mathrm{Cl} A=A \cup \operatorname{Int} \mathrm{Cl} A$ and $\operatorname{sIn} t A=A \cap \mathrm{Clnt} A[15]$. A space $(X, \tau)$ is extremally disconnected iff $\mathrm{ClG} \in \tau$ for every $G \in \tau$. A space $(X, \tau)$ is said to be quasi $H$-closed [12] (nearly compact [14], s-closed [16]) iff for every cover $\left\{V_{\alpha}: a \in \Delta\right\}$ of $X$ such that $V_{\alpha} \in \tau$ (resp. $\nabla_{a} \in \tau, V_{a} \in S O(X, \tau)$ ) for all $a \in \Delta$, there exists a finite subset $\Delta_{0}$ of $\Delta$ such that $X=\bigcup_{a \in \Delta_{0}} \mathrm{ClV} V_{a}$ (resp. $X=\bigcup_{\alpha \in \Delta_{0}} \operatorname{IntClV_{a}}, X=\bigcup_{a \in \Delta_{0}} \mathrm{ClV} V_{a}$ ). A space $(X, \tau)$ is called almost regular [13] ( $s$-regular [7]) iff for each $G \in R O(X, \tau)$ (resp. $G \in \tau$ ) containing a point $x \in X$ there exists $U \in \tau($ resp. $U \in S O(X, \tau))$ such that $x \in U \subset \mathrm{ClU} \subset G$ (resp. $x \in U \subset s \mathrm{C} l U \subset G$ ).

\section{Regular Semi-Open Sets}

Tyefinition 2.1. A subset $A$ of a space $(X, \tau)$ is said to be a regular wemi opes: set [2] iff $A=\operatorname{sints} \mathrm{C} / \mathrm{A}$. We shall dencte the class of all regular smi- open subsets of a space $(X, \tau)$ by $R S O(X, \tau)$. It is clear that $R S O(X, \tau) \subset$ S) $(\mathrm{r}, \tau)$.

Lemma 2.1. For any subset $A$ of a space $(X, \tau)$.

\section{$\operatorname{IntCl} A \subset \operatorname{sints} \mathrm{Cl} A \subset \mathrm{C} l \operatorname{Int} \mathrm{Cl} A$}

Proof. Straightforward.

Lerma 2.2. Let $(X, \tau)$ be any space. Then the following statements are quivalent

(i) $A \in R S O(X, \tau)$.

(ii) $X \backslash A \in R S O(X, \tau)$.

(iii) $A=s C l s \operatorname{Int} A$.

(iv) $A \in S O(X, \tau) \cap S C(X, \tau)$.

(v) there exists $U \in R O(X, \tau)$ such that $U \subset A \subset C l U$.

Proof. (i) $\rightarrow$ (ii) Let $A \in R S O(X, \tau)$. Then $A=\operatorname{sint} s \mathrm{Cl} A$. By lemma 2.1, wo deduce that $\operatorname{IntCl} A \subset A$, i.e. $A \in S C(X, \tau)$ and hence $X \backslash A=\operatorname{sints} \mathrm{Cl}(X \backslash A)$. 
Consequently, $X \backslash A \in R S O(X, \tau)$.

(ii) $\rightarrow$ (iii). Obvious.

(iii) $\rightarrow$ (iv). Obvious.

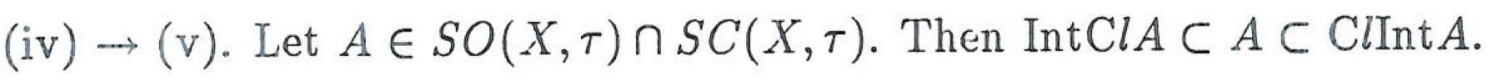

Taking $U=\operatorname{IntCl} A \in R O(X, \tau)$, we get

\section{$U \subset A$ and $A \subset \mathrm{C} l \operatorname{Int} A \subset \mathrm{ClIntC} l A=\mathrm{ClU}$,}

i.e. there exists $U \in R O(X, \tau)$ such that $U \subset A \subset \mathrm{ClU}$.

$(\mathrm{v}) \rightarrow(\mathrm{i})$. Let $U \in R O(X, \tau)$ such that $U \subset A \subset \mathrm{Cl} A$. Then $\mathrm{ClU}=\mathrm{Cl} A$, $U=\operatorname{Int} C l U=\operatorname{IntCl} A$ and so $\operatorname{IntCl} A \subset A$. Now, since $U \subset \operatorname{Int} A$ and $\operatorname{Int} A \subset$ $\operatorname{IntCl} A=U$, so $U=\operatorname{Int} A$ and $C l U=C l I n t A$, which implies that $A \subset \mathrm{C} l \operatorname{Int} A$. Accordingly,

$$
\begin{aligned}
& s \operatorname{Int} s \mathrm{C} l A=s \mathrm{C} l \mathrm{~A} \cap \mathrm{C} / \ln \mathrm{t} s \mathrm{C} / \mathrm{A} \\
& =(A \cup \operatorname{Int} C l A) \cap C l \operatorname{Int}(A \cup \operatorname{IntCl} A) \\
& =A \cap \operatorname{Cln} \operatorname{A}=A \text {, }
\end{aligned}
$$

i.e. $A \in R S O(X, \tau)$. This completes the proof of the lemma.

Lemma 2.3. For any space $(X, \tau)$,

$$
R O(X, \tau) \cup R C(X, \tau) \subset R S O(X, \tau) .
$$

Proof. If $A \in R O(X, \tau)$ or $A \in R C(X, \tau)$, then $A \in S O(X, \tau)$ and $A \in$ $S C(X, \tau)$, which implies that $A \in R S^{\prime} O(X, \tau)$ by lemma 2.2 .

Example 2.1. The inclusion relation in lemma 2.3 , in general, cannot be replaced by equality. As an example let $X=\{a, b, c, d\},, \tau=\{X, \phi,\{a\},\{b\}$, $\{a, b\},\{a, b, c\}\}$. Then $\{a, c\} \in R S O(X, \tau)$ but $\{a, c\} \notin R O(X, \tau) \cup R C(X, \tau)$.

We have the following diagram of implications, and any other, except those 
resulting by transitivity, can not be added, in general.

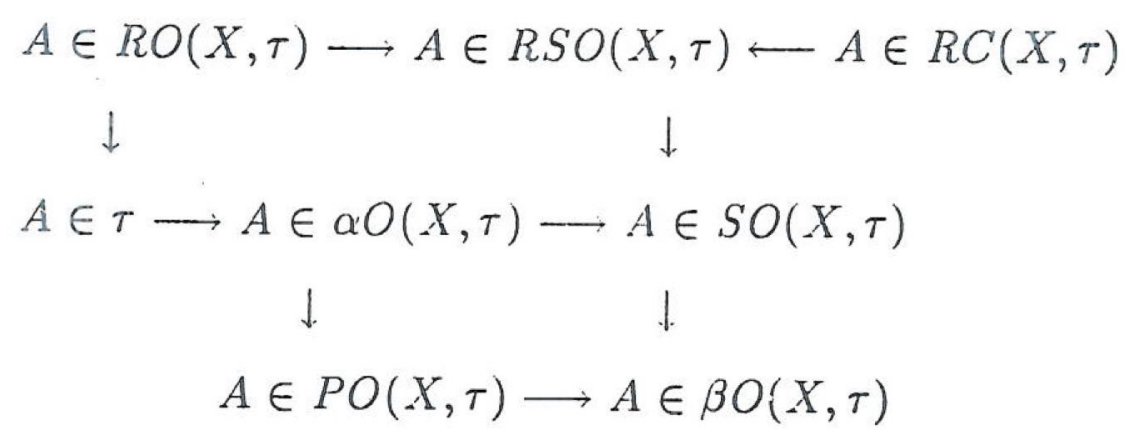

As a sample we give the following example.

Example 2.2 Example 2.1 shows that

(i) $\tau$ and $R S O(X, \tau)$ may be not comparable, in general.

(ii) $\alpha O(X, \tau)$ and $R S O(X, \tau)$ may be not comparable, in general.

(iii) $R O(X, \tau) \neq R S O(X, \tau) \neq R C(X, \tau)$, in general.

(iv) $R S O(X, \tau)$, may be neither supratopology nor infratopology [5] on $\mathrm{X}$, in general.

Lemma 2.4. For any space $(X, \tau)$, if $A \in R S O(X, \tau)$ and $A \subset B \subset \mathrm{Cl} A$, then $B \in R S O(X, \tau)$.

Proof. By lemma 2.2, there exists $U \in R O(X, \tau)$ such that $U \subset A \subset \mathrm{ClU}$. Hence, $U \subset B \subset \mathrm{Cl} U$ and $B \in R S O(X, \tau)$ by using lemma 2.2 again.

Lemma 2.5. For any space $(X, \tau), A \in R S O(X, \tau)$ iff there exist an open set $G$ and a closed set $F$ such that

$$
\operatorname{Int} F \subset G \subset A \subset F \subset \mathrm{Cl} G
$$

Proof. Straightforward.

Lemma 2.6. For any space $(X, \tau)$,

$$
P O(X, \tau) \cap R S O(X, \tau)=R O(X, \tau)
$$


Proof. Suppose that $A \in P O(X, \tau)$ and $A \in R S O(X, \tau)$. Then $A \subset \operatorname{IntCl} A$ and $A=s \operatorname{Int} \mathrm{Cl} A$. Therefore,

$$
A=s \operatorname{Int}(A \cup \operatorname{IntCl} A)=s \operatorname{Int} \operatorname{Int} C l A=\operatorname{IntCl} A,
$$

i.e. $A \in R O(X, \tau)$. The reverse implication follows directly from the above diagram.

Corollary 2.1. For any space $(X, \tau)$,

$$
P C(X, \tau) \cap R S O(X, \tau)=R C(X, \tau)
$$

Proof. Obvious.

Remarks. Lemma 2.6 and corollary 2.1 may be not true in general even if $A \in \beta O(X, \tau)$ (resp. $A \in \beta C(X, \tau)$ ) instead of $A \in P O(X, \tau)$ (resp. $A \in$ $P C(X, \tau))$. In example 2.1, $\{a, c\} \in \beta O(X, \tau) \cap R S O(X, \tau)$, but $\{a, c\} \notin$ $R O(X, \tau)$.

Lemma 2.7. For each subset $A$ of a space $(X, \tau)$, the sets $\operatorname{sints} \mathrm{Cl} A$, Ints $\mathrm{Cl} A$, sIntClA and $\operatorname{Int} \mathrm{Cl} A$ are rgular semi-open sets.

Proof. We shall prove that $\operatorname{sints} \mathrm{Cl} A \in R S O(X, \tau)$ and the proof of the other parts is then obvious. We have by lemma 2.1

$$
\text { Int } C l A \subset \operatorname{sints} C l A \subset C l \operatorname{In} l \mathrm{C} l A \text {, }
$$

where IntClA $\in R O(X, \tau)$. The result follows directly by using lemma 2.2.

Corollary 2.2. The closure and the semi-closure of a semi-open subset A of a space $(X, \tau)$ are regular semi-open.

Proof. Since $A \in S O(X, \tau)$, so $\mathrm{Cl} A=\mathrm{C} l \operatorname{Int} A$ and $s \mathrm{Cl} A \subset \mathrm{C} l \operatorname{Int} s \mathrm{Cl} A$. Therefore, $\mathrm{Cl} A, s \mathrm{Cl} A \in S O(X, \tau)$. But $\mathrm{Cl} A, s \mathrm{C} l A \in S C(X, \tau)$, hence the result follows by lemma 2.2 . 
Corollary 2.3. For each subset $A$ of a space $(X, \tau)$, the sets $\operatorname{Cln} \operatorname{Int} A$, $\mathrm{Cls} \operatorname{Int} A, s \mathrm{ClInt} A$ and $s \mathrm{Cls} \operatorname{Int} A$ are regular semi-open.

Proof. This follows directly by corollary 2.2 and by observing that $\operatorname{Int} A$, $\operatorname{sint} A \in S O(X, \tau)$.

Lemma 2.8. For any space $(X, \tau)$, if $Y \in \alpha O(X, \tau)$ and $A \in R S O(X, \tau)$ then $A \cap Y \in R S O\left(Y, \tau_{Y}\right)$.

Proof. Since $A \in R S O(X, \tau)$, so $A \in S O(X, \tau)$ and $X \backslash A \in S O(X, \tau)$. Therefore, $A \cap Y \in S O\left(Y, \tau_{Y}\right)$ and $(X \backslash A) \cap Y \in S O\left(Y, \tau_{Y}\right)[9]$. But ( $X \backslash$ $A) \cap Y=Y \backslash(A \cap Y)$ and hence $Y \backslash(A \cap Y) \in S O\left(Y, \tau_{Y}\right)$. Consequently, $A \cap Y \in R S O\left(Y, \tau_{Y}\right)$.

Lemma 2.8 may be not true, in general, even if $Y \in R S O(X, \tau)$, as the following example shows.

Example 2.3. Taking $Y=\{a, c, d\}$ and $A=\{b, c, d\}$ in example 2.1, then $A \cap Y \notin R S O\left(Y, \tau_{y}\right)$.

Lemma 2.9. For any space $(X, \tau)$, if $Y \in R O(X, \tau)$ and $A \in R S O\left(Y, \tau_{y}\right)$, then $A \in R S O(X, \tau)$.

Proof. By lemma 2.2, we have $A \in S O\left(Y, \tau_{y}\right)$ and $Y \backslash A \in S O\left(Y, \tau_{y}\right)$. This implies that $A \in S O(X, \tau)$ and $Y \backslash A \in S O(X, \tau)$ [10]. Since $Y$ is regular open in $X$, so it is regular semi-open in $X$ and hence $X \backslash Y \in S O(X, \tau)$. Therefore, $(Y \backslash A) \cup(X \backslash Y)=X \backslash A \in S O(X, \tau)$. Accordingly, $A \in S C(X, \tau)$. By lemma 2.2, $A \in R S O(X, \tau)$.

3. $S^{*}-$ Closed Spaces

Definition 3.1. A filterbase $\mathcal{F}$ in a space $(X, \tau) s^{*}$-converges to a point $x_{0} \in X$ iff for each $A \in R S O(X, \tau)$ such that $x_{0} \in A$, there exists an $F \in \mathcal{F}$ such that $F \subset A$. 
Definition 3.2. A filterbase $\mathcal{F}$ in a space $(X, \tau) s^{*}$-accumulates to $x_{0} \in X$ iff for each $A \in R S O(X, \tau)$ such that $x_{0} \in A$ and each $F \in \mathcal{F}, F \cap A \neq \phi$.

The following lemma is an easy consequence of the above definitions.

Lemma 3.1. If $\mathcal{F}$ is a maximal filterbase in a space $(X, \tau)$, then $\mathcal{F} s^{*}$-accunmulates to $x_{0} \in X$ iff $\mathcal{F} s^{*}$-converges to $x_{0}$.

Theorem 3.1. A filterbase $\mathcal{F}$ in a space $(X, \tau) s^{*}$-converges to $x_{0} \in X$ iff for each $A \in S O(X, \tau)$ such that $x_{0} \in A$, there exists an $F \in \mathcal{F}$ such that $F \subset s C l A$.

Proof. (Necessity). Suppose that $\mathcal{F} s^{*}$-converges to $x_{0} \in X$ and $A \in$ $S O(X, \tau)$ such that $x_{0} \in A$. By corollary $2.2, s \mathrm{ClA} \in R S O(X, \tau)$ and $x_{0} \in s \mathrm{ClA}$. Therefore, there exists an $F \in \mathcal{F}$ such that $F \subset s \mathrm{C} l A$.

(Sufficiency). Let the condition be satisfied and let $A \in R S O(X, \tau)$ such that $x_{0} \in A$. Then $A \in S O(X, \tau)$ and therefore, there exists an $F \in \mathcal{F}$ such that $F \subset s C l A$. By lemma 2.2, $A \in S C(X, \tau)$. Consequently, $s \mathrm{Cl} A=A$ and the proof is complete.

The following theorem can be proved similarly.

Theorem 3.2. A filterbase $\mathcal{F}$ in a space $(X, \tau) s^{*}$-accumulates to $x_{0} \in X$ iff for each $A \in S O(X, \tau)$ such taht $x_{0} \in A$ and each $F \in \mathcal{F}, F \cap s \mathrm{Cl} A \neq \phi$.

Definition 3.3. A space $(X, \tau)$ is said to be $s^{*}$-closed space iff each regular semi-open cover of $X$ has a finite subcover.

Theorem 3.3. Each $s^{*}$-closed space is nearly compact and $s$-closed.

Proof. Obvious.

It is known that the one point compactification of a finite discrete space is not $s$-closed [16], and by theorem 3.3, is therefore not $s^{*}$-closed. But it is nearly compact.

Theorem 3.4. A space $(X, \tau)$ is $s^{*}$-closed iff for each semi-open cover 
$\left\{A_{a}: a \in \Delta\right\}$ of $X$, there exists a finite subset $\Delta_{0}$ of $\Delta$ such that $X=$ $\bigcup_{a \in \Delta_{0}} s \mathrm{Cl} A_{a}$.

Proof. (Necessity). Let $(X, \tau)$ be $s^{*}$-closed and $\left\{A_{a}: a \in \Delta\right\}$ be a semi-open cover of $X$. By corollary 2.2, we have $\operatorname{sCl} A_{a} \in R S O(X, \tau)$ for every $a \in \Delta$ and $X=\bigcup_{a \in \Delta} s \mathrm{Cl} A_{a}$. Therefore, there exists a finite subset $\Delta_{0}$ of $\Delta$ such that $X=\bigcup_{a \in \Delta_{0}} s \mathrm{Cl} A_{a}$.

(Sufficiency). Let $\left\{A_{a}: a \in \Delta\right\}$ be any regular semi-open cover of $X$. Then, by lemma 2.2, $A_{a} \in S O(X, \tau)$ and $A_{a} \in S C(X, \tau)$, for each $a \in \Delta$. Therefore, by the hypothesis, there exists a finite subset $\Delta_{0}$ of $\Delta$ such that

$$
X=\bigcup_{a \in \Delta_{0}} s \operatorname{Cl} A_{a}=\bigcup_{a \in \delta_{0}} A_{a}
$$

which shows that $(X, \tau)$ is $s^{*}$-closed.

Theorem 3.5. For any space $(X, \tau)$, the following statements are equivalent.

(i) $(X, \tau)$ is $s^{*}$-closed.

(ii) For each semi-open cover $\left\{A_{a}: a \in \Delta\right\}$ of $X$, there exists a finite subset $\triangle_{0}$ of $\Delta$ such that $X=\bigcup_{a \in \Delta_{0}} s \mathrm{Cl} A_{a}$.

(iii) For each family $\left\{F_{a}: a \in \Delta\right\}$ of semi-closed subsets of $X$ such that $\bigcap_{a \in \Delta} F_{a}=\phi$, there exists a finite subset $\Delta_{0}$ of $\Delta$ such that $\bigcap_{a \in \Delta_{0}} F_{a}=\phi$. (iv) for each family $\left\{F_{a}: a \in \Delta\right\}$ of semi-closed subsets of $X$, if $\bigcap_{a \in \Delta_{0}}^{a \in \Delta_{0}} \operatorname{sint} F_{a} \neq \phi$ for every finite subset $\Delta_{0}$ of $\Delta$, then $\bigcap_{a \in \Delta} F_{a} \neq \phi$.

(v) for each family $\left\{A_{a}: a \in \Delta\right\}$ of regular semi-open subsets of $X$ such that $\bigcap_{a \in \Delta} A_{a}=\phi$, there exists a finite subset $\Delta_{0}$ of $\Delta$ such that $\bigcap_{a \in \Delta_{0}} A_{a}=\phi$. (vi) for each family $\left\{A_{a}: a \in \Delta\right\}$ of regular semi-open subsets of $X$, if $\bigcap_{a \in \Delta_{0}} A_{a}$ $\neq \phi$ for every finite subset $\Delta_{0}$ of $\Delta$, then $\bigcap_{a \in \Delta} A_{a} \neq \phi$.

(vii) Every filterbase $\mathcal{F}$ in $X s^{*}$-accumulates to some point $x_{0} \in X$.

(viii) Every maximal filterbase $\mathcal{F}$ in $X s^{*}$-converges to some point $x_{0} \in X$. 
Proof. By theorem 3.4 we have (i) $\leftrightarrow$ (ii) and the equivalencies (iii) $\leftrightarrow$ (iv) and $(\mathrm{v}) \leftrightarrow$ (vi) are obvious.

(ii) $\rightarrow$ (iii). Let $\left\{F_{a}: a \in \Delta\right\}$ be a family of semi-closed subsets of $X$ such that $\bigcap_{a \in \Delta} F_{a}=\phi$. Therefore, $X=\bigcup_{a \in \Delta}\left(X \backslash F_{a}\right)$, where $X \backslash F_{a} \in S O(X, \tau)$ for all $a \in \Delta$. By (ii), there exists a finite subset $\Delta_{0}$ of $\Delta$ such that

$$
X=\bigcup_{a \in \Delta_{0}} s \operatorname{Cl}\left(X \backslash F_{a}\right)=\bigcup_{a \in \Delta_{0}}\left(X \backslash s \operatorname{Int} F_{a}\right)=X \backslash\left(\bigcap_{a \in \Delta_{0}} s \operatorname{Int} F_{a}\right),
$$

which implies that $\bigcap_{a \in \Delta_{0}} s \operatorname{Int} F_{a}=\phi$.

(iii) $\rightarrow(v)$. Let $\left\{A_{a}: a \in \Delta\right\}$ be a family of regular semi-open subsets of $X$ such that $\bigcap_{a \in \Delta} A_{a}=\phi$. By lemma. $2.2, A_{a} \in S O(X, \tau) \cap S C(X, \tau)$ for all $a \in \Delta$. Using (iii), there exists a finite subset $\Delta_{0}$ of $\Delta$ such that $\bigcap_{a \in \Delta_{0}} \operatorname{sint} A_{a}=\phi=$ $\bigcap_{a \in \Delta_{0}} A_{a}$

$(\mathrm{v}) \rightarrow(\mathrm{i})$. Let $\left\{A_{a}: a \in \Delta\right\}$ be any regular semi-open cover of $X$. Therefore, $\bigcap_{a \in \Delta}\left(X \backslash A_{a}\right)=\phi$ and by lemma 2.2, $X \backslash A_{a} \in R S O(X, \tau)$ for all $a \in \Delta$. Using (v), there exists a finite subset $\Delta_{0}$ of $\Delta$ such that $\bigcap_{a \in \Delta_{0}}\left(X \backslash A_{a}\right)=\phi$. This implies that $\bigcup_{a \in \Delta_{0}} A_{a}=X$ and $X$ is $s^{*}$-closed.

(i) $\rightarrow$ (viii). Suppose that $\mathcal{F}=\left\{F_{a}: a \in \Delta\right\}$ is a maximal filterbase in $X$ which does not $s^{*}$-converge to any point in $X$. Therefore, by lemma $3.1, \mathcal{F}$ does not $s^{*}$-accumulate to any point in $X$. This implies that for every $x \in X$, there exist $A(x) \in R S O(X, \tau)$ containing $x$ and $F_{a(x)} \in \mathcal{F}$ such that $F_{a(x)} \cap A(x)=\phi$. Hence, the family $\{A(x): x \in X\}$ is a regular semi-open cover of $X$ and by the hypothesis, there is a finite subfamily $\left\{A\left(x_{i}\right): i=1,2, \ldots, n\right\}$ such that $X=\bigcup_{i=1}^{n} A\left(x_{i}\right)$. Since $\mathcal{F}$ is a filterbase in $X$, there exists an $F_{0} \in \mathcal{F}$ such that $F_{0} \subset \bigcap_{i=1}^{n} F_{a\left(x_{i}\right)}$. Accordingly, $F_{0} \cap A\left(x_{i}\right)=\phi$ for all $i \in\{1,2, \ldots, n\}$. This implies that

$$
\phi=F_{0} \cap\left(\bigcup_{i=1}^{n} A\left(x_{i}\right)\right)=F_{0} \cap X,
$$


i.e. $F_{0}=\phi$. This is a contradiction and consequently, $\mathcal{F}$ must $s^{*}$-converges to some point $x_{0} \in X$.

(viii) $\rightarrow$ (vii). Follows directly from lemma 3.1 and the fact that every filterbase is contained in a maximal filterbase.

(vii) $\rightarrow(\mathrm{v})$. Let $\left\{A_{a}: a \in \Delta\right\}$ be a family of regular semi-open subsets of $X$ such that $\bigcap_{a \in \Delta} A_{a}=\phi$. Suppose that for every finite subfamily $\left\{A_{a_{i}}: i=\right.$ $1,2, \ldots, n\}, \bigcap_{i=1}^{n} A_{a_{i}} \neq \phi$. Therefore,

$$
\mathcal{F}=\left\{\bigcap_{i=1}^{n} A_{a_{i}}: n \in N, \quad a_{i} \in \Delta\right\}
$$

forms a filter base in $X$. Using (vii), $\mathcal{F} s^{*}$-accumulates to some point $x_{0} \in X$. This implies that, for every $A\left(x_{0}\right) \in R S O(X, \tau)$ containing $x_{0}, F \cap A\left(x_{0}\right) \neq \phi$ for every $F \in \mathcal{F}$. Since $\bigcap_{F \in \mathcal{F}} F=\phi$, there exists $F_{0} \in \mathcal{F}$ such that $x_{0} \notin F_{0}$, which implies that there exists $a_{0} \in \Delta$ such that $x_{0} \notin A_{a_{0}}$. Accordingly, $x_{0} \in X \backslash A_{a_{0}}$ and $X \backslash A_{a_{0}} \in R S O(X, \tau)$ by using lemma 2.2. Therefore, there exists $a_{0} \in \Delta$ such that $F_{0} \cap\left(X \backslash A_{a_{0}}\right)=\phi$ contradicting the fact that $\mathcal{F} s^{*}$-accumulates to $x_{0}$. This completes the prool.

Theorem 3.6. Each $s$-regular and $s^{*}$-closed space is compact.

Proof. Let $\left\{G_{a}: a \in \Delta\right\}$ be any open cover of an $s$-regular and $s^{*}$-closed space $(X, \tau)$. Then for each $x \in X$, there exists an $a(x) \in \Delta$ such that $x \in G_{a(x)}$. Since $(X, \tau)$ is $s$-regular, there exists $A(x) \in S O(X, \tau)$ such that $x \in A(x) \subset s \mathrm{Cl} A(x) \subset G_{a(x)}$. Therefore, the family $\{s \mathrm{Cl} A(x): x \in X\}$ is a regular semi-open cover of $(X, \tau)$ (by using corollary 2.2). Since $(X, \tau)$ is $s^{*}$-closed, there exists a finite subfamily $\left\{A\left(x_{i}\right): i=1,2, \ldots, n\right\}$ such that $X=\bigcup_{i=1}^{n} s \mathrm{Cl} A\left(x_{i}\right) \subset \bigcup_{i=1}^{n} G_{a\left(x_{i}\right)}$, which completes the proof.

The following example shows that the condition of $s$-regularity in theorem 3.6 can not be dropped.

Example 3.1. Let $X=(0,1)$ with the topology $\tau$ consisting of $X, \phi$ and 
all subsets of $X$ of the form $(0,1-1 / n)$, where $n=2,3, \ldots$ Then $(X, \tau)$ is neither $s$-regular nor compact, but it is $s^{*}$-closed because the only non-empty regular semi-open set in $(X, \tau)$ is $X$ itself.

Using theorem 3.4 and lemma 4.1 of [11], we get the following result.

Corollary 3.1. Each extremally disconnected $s$-closed space is $s^{*}$-closed.

Using corollary 3.1 and theorem 3.4 of [4], we get the following reslut.

Corollary 3.2. If a spase $(X, \tau)$ is nearly compact (or quasi $H$-closed) and extremally disconnected, then $(X, \tau)$ is $s^{*}$-closed.

Using corollary 3.1 and theorem 3.5 of [4], we get the following result.

Corollary 3.3. Each almost regular and $s$-closed space is $s^{*}$-closed.

\section{References}

[1] M. E. Abd El-Monsef, S. N. El-Deeb and R. A. Mahmoud, " $\beta$-open sets and $\beta$-continuous mappings", Bull. Fac. Sci. Assiut Univ. (1983) 1-18.

[2] D. E. Cameron, "Properties of s-closed spaces", Proc. Amer. Math. Soc., 72 (1978), 581-586.

[3] S. G. Crossley and S. K. Hildebrand, "Semi-closure, Taxas", J. Sci. $22(2+3)$ (1971), 99-112.

[4] R. A. Herman, "RC-convergence", Proc. Amer. Math. Soc., 75 (1979), 311-317.

[5] T. Husain, "Topology and maps", Plenum Press, 1977.

[6] N. Levine, "Semi-open scts and semi-continuity in topological spaces", Amer. Math. Monthly, 70 (1963) 36-41 MR 29 \# 4025.

[7] S. N. Maheshwari and R. Prasad, "On s-regnlar spaces", Glasnik Mat. Ser. III 10(30) (1975), 347-350.

[8] A. S. Mashour, M. E. Abd El-Monsel and S. N. El-Deeb, "On pre-continuous and week pre-continuous mappings", Proc. Math. Phys. Soc. Egypt 53 (1982), 47-53.

[9] O. Njastad, "On some classes of nearly open sets", Pacific J. Math. 15 (1965) 961-970.

[10] T. Noiri, "On semi-continuous mappings", Atti. Accad. Naz. Lincei Rend. Cl. Sci. Fiz. Mat. Natur 8 (54) (1973), 210-214.

[11] T. Noiri, "Properties of s-closed spaces", Acla Math. Acad. Sci. Hung. 53 (3-4) (1980), $431-436$.

[12] J. Porter and J. Thomas, "On $H$-closed and minimal Hausdorff spaces", Trans. Amer. Math. Soc. 138 (1969), 159-170.

[13] M. K. Singal S. P. Arya, "On almost regular spaces", Glasnik Mat. Ser. III (4) 24 (1969), 89-99. 
[14] M. K. Singal and A. Mathur, "On nearly compact spaces", Boll. Un. Math. Ital., (4) 2 (1969), 702-710.

[15] S. F. Tadros and A. S. Abd Allah, "On some mappings of topological spaces", Accepted in Ain Shams Univ. Sci. Bull.

[16] T. Thompson, "S-closed spaces", Proc. Amer. Math. Soc., 60 (1976), 335-338.

Department of Mathematics, Faculty of Science, University of Mansoura, Mansoura, EGYPT. 